Historic, Archive Document

Do not assume content reflects current scientific knowledge, policies, or practices. 
Prices quoted are for extra strong plants; 5 per rate of 10 ; single plants postage prepaid. Terms cash.

Each Ten 100

ADMIRAL TOGO: S. \& F. white bordered lavender, same habit as Mad Chereau, extra fine 36inch......\$.20 $\$ .80$

ALBERT VICTOR: S. \& F. soft lavender blue, vigorous grower, one of the best of the Pallida section, 48-inch

BLACK PRINCE: S. Purple Lilac; F. velvety black, an early flowering variety.

CELESTA: S. \& F. Pale azure blue, one of the most beautiful

CYPRIANA: S. Pale blue, F. long, rich purple resembles Pallida

DR. BERNICE: S. Coppery bronze; F. velvety crimson, large and beautiful. Extra fine 24-inch..

GERTRUDE: S. \& F. Violet blue, an early free bloomer; 32-inch

HARLEQUIN MELANOIS: S. \& F. white and blue; 32-inch; one of the most curious mottled of all irises

INGEBORG: S. \& F. pure white, large flowers, the best white in the trade; fine for cut flowers; 24-inch

INNOCENZA: S. \& F. ivory white, crest rich golden; a fine white variety.

IRIS KING: S. lemon yellow; F. maroon bordered yellow; a very beautiful new variety; 34 -inch........ .30

ISOLINE: S. lilac pink; F. purplish rose; one of the grandest varieties in cultivation, 36 -inch..

JACQUESIANA: S. bright copper crimson;.F. bright maroon; distinct one of the best reds, 36-inch..

JOHN De WERTH: S. light mauve; F. purple heavily veined, 30 -inch

HER MAJESTY: S. rose pink; F. crimson, tinged with darker shade, 32-inch..

LEONIDAS: S. violet blue; F. rich velvety purple, late flowering, 26-inch.

MACRANTHA: (Syn. Amas) S. rich blue; F. violet, early flowering, extra fine and large, 24-inch...... 
MAD CHEREAU: S. \& F. white frilled with a wide border of clear blue, extra fine, 34-inch.

MAD P'AQUETTE: S. \& F. bright rosy red, distinct flowers produced freely

OTHELLO: S. deep rich blue; $F$. dark velvety purple, very fine, 26 -inch

PAL. DALMATICA: S. deep lavender; F. shaded light purple, a grand variety, large flowers.

PAL. MANDRALISCOE: F. \& S. rich lavender purple, tall, large and handsome, 40-inch

PAL. SPECIOSA: S. dark lavender shaded lighter; F. shaded light purple.

PALLIDA: S. \& F. lavender blue. This variety was bought over 50 years ago as Agathocles. The strongest grower of this section, leaves 2 inches broad, 48-inch

PARISENSIS: S. purple lilac; F. rich velvety blue, very early, 26-inch

PERFECTION : S. light blue; F. dark velvety violet, a handsome abundant bloomer, 32-inch.

PRINESS VICTORIA: S. sulphur yellow; F. plum color, bordered cream, extra fine, 32 -inch.

QUEEN of MAY: S. \& F. soft rosy lilac shaded pink, a free bloomer

RHEINNIXE: S. pure white; F. violet blue with an edge, very handsome, extra fine, 32-inch

TAMERLANE: S. pale purple; F. deep violet purple, very large, a handsome variety, 3 -inch.

The following new varieties I can supply only in single plants as they did not increase any on account of the drought.

ARCHEVEQUE: S. rich purple; $F$. deep velvety violet; extreme rich coloring, 24-inch.

CAPRICE: reddish purple; $F$. deeper purple, large and beautiful, 36 -inch

CATERINA: Claimed to be an improved Dalmatica; a high priced iris, like to rot................................. ... .75

ELDORADO: S. bronze shaded yellow; F. purple bordered yellow 
Each Ten 100

LOHENGRIN : S. \& F. light rose, large and handsome, vigorous grower $\$ .75$

MONSIGNOR: S. lavender; F. velvety purple crimson,

a large handsome variety, 24-inch............................. .75

PRINCESS VICTORIA: S. \& F. pure white, 32-inch..... 50

QUEEN EMMA: S. \& F. clear waxy white................... .50

TWILIGHT: S. \& F. delicate violet purple................... .50

VICTORINE: S. white; F. mottled violet........................ .30

WYOMISSING: S. rosy white; F. deep rose shading

to flesh .30

\section{SIBERIAN IRIS}

The Siberian Irisis have narrow grassy foliage with beautiful flowers. Fine for cutting.

SIBERIAN BLUE $\$ .25$

SIBERIAN GRANDIS: Violet reticulated, white.

SIBERIAN ORIENTALS: Deep blue, large for its class .25

SIBERIAN SUPERBIA: Violet blue, handsome foliage.. .25

All orders are accepted on condition, that I am not sold out of varieties wanted, and they shall be roid, should injury befall my stock from frost, fire, or other cause, over which I have no control. All varieties are guaranteed true to name. My Gladioli and Dahlia catalogue will be issued next December.

HENRY C. ECKERT

Belleville, Ill.

\section{THE FLOWER GROWER}

(Formerly the Modern Gladiolus Grorrer)

Published Monthly by Madison Cooper at Calcium, N. Y.

Subscription Price: Three Years, $\$ 2.00$; One Year, $\$ 1.00$. 